\title{
Performance Evaluation and Comparison of Different Noise, apply on PNGImage Format used in Deconvolution Wiener filter (FFT) Algorithm
}

\author{
Kalpana Chaurasia and Mrs. Nidhi Sharma* \\ Dept. of ECE, M.P.C.T. Gwalior, M.P., India. \\ Email: Kalpana.chaurasia86@gmail.com
}

Keywords: FastfourierTransform, MSE, PSNR, RMSE, MAE.

\begin{abstract}
Image Restoration is a field of Image Processing. This deals with recovering an original and sharp image from a degraded image using degradation \& restoration function. This study focus on restoration of degraded images which have been blurred by known degradation function.PNG(Tag Index Format) are considered for analyzing the image restoration techniques deconvolution using wiener filter(FFT) algorithm with an information of the Point Spread Function (PSF) corrupted blurred image and then corrupted by Different noise. Performance analysis is done to measure the efficiency by which image is recovered. The analysis is done on the basis of various performance metrics like Peak Signal to Noise Ratio(PSNR), Mean Square Error(MSE), Root Mean Square Error(RMSE), Mean Absolute Error(MAE).
\end{abstract}

\section{INTRODUCTION}

Image restoration techniques can improve the apparent sharpness of image from the degraded image using degradation \& restoration model. Image capture process Causes degradation of original image. There are several factors having contributions to the blur, two of them are the most important[1]:-Blur occur due to both object or camera motion is called motion blur.[2]out of focus optic caused by wide angle lens setting or atmospheric turbulence, being called out of focus blur. Degraded image is additionally corrupted by the noise. The noise is a consequence of imperfection of image sensor and acquisition part of camera. In Degradation model, the original image is blurred using degradation function and noise. Degradation image can be described by the formula:

$$
\mathrm{g}=\mathrm{f} * \mathrm{~h}+\mathrm{n}
$$

Where:-g is degraded image is the degradation function is an original image and $\mathrm{n}$ is the noise.

The Degradation model is structure as follows:

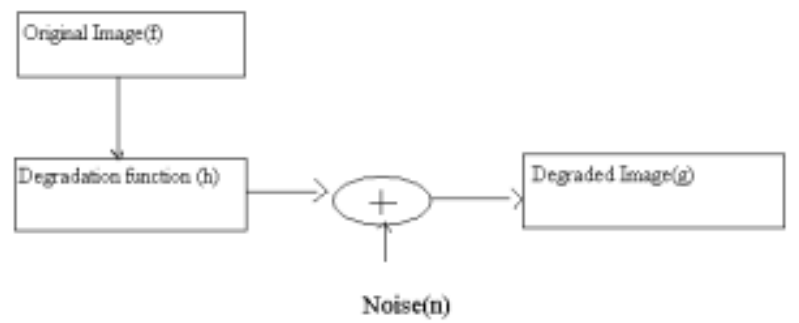

Fig.1 Degradation Model

Image deconvolution (sometimes known as image deblurring) is the process of reconstructing or estimating the true image from the degraded one. In Restoration Model, the degraded image is regenerate using Deconvolution wiener filter (FFT) algorithm. It performs the inverse process of degradation by removing noise and blur factor. We get an estimate of the original image as a restoration result. 


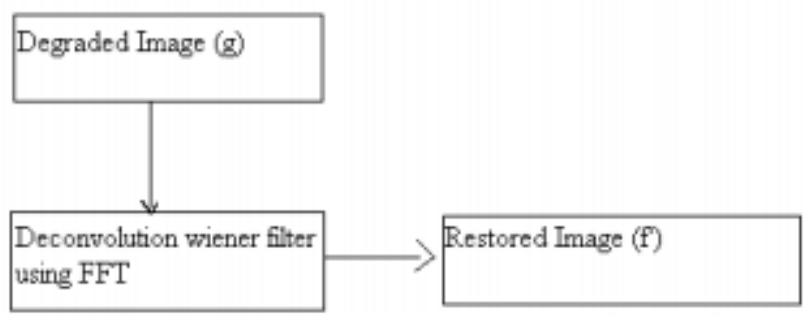

Fig.2 Restoration model

\section{DECONVOLUTION USING WIENER FILTER (FFT TECHNIQUE)}

The wiener filter is an image restoration technique for the deconvolution with a priori known PSF.The wiener filter is usually applied in the frequency domain. given a blurred image $g(x, y)$.one takes the discrete Fourier transform (DFT)to obtain G(u,v).the original image spectrum is estimated by taking the product of $\mathrm{G}(\mathrm{u}, \mathrm{v})$ with the wiener filter $\mathrm{W}(\mathrm{u}, \mathrm{v})$ :

$$
\mathrm{F}(\mathrm{u}, \mathrm{v})=\mathrm{G}(\mathrm{u}, \mathrm{v}) \mathrm{w}(\mathrm{u}, \mathrm{v})
$$

The inverse DFT is then used to obtain the original image from its spectrum. The Wiener filter can be stated as follows:

$$
\mathrm{W}(\mathrm{u}, \mathrm{v})=\mathrm{H}^{*}(\mathrm{u}, \mathrm{v}) / /\left.\mathrm{H}(\mathrm{u}, \mathrm{v})\right|^{2}+\mathrm{P}
$$

Where $\mathrm{H}(\mathrm{u}, \mathrm{v})$ denote Fourier Transform of the PSF .Since the $\mathrm{w}(\mathrm{u}, \mathrm{v})$ have the possibility to produce the pulse while the $|\mathrm{H}(\mathrm{u}, \mathrm{v})|^{2}$ approaches zero. Hence, in adopted $\mathrm{P}$ to prevent this situation.

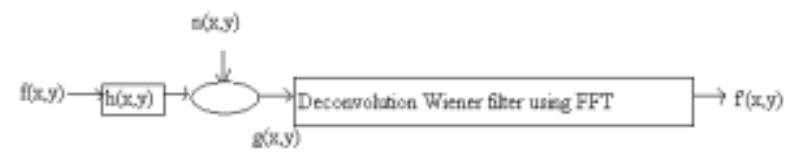

Fig.3 Deconvolution using wiener filter (FFT technique)

\section{a) Methodology}

This paper aims at studying png image formats used in Deconvolution using wiener filter (FFT) Technique. We will first degraded the original image using a blur and different noise then by using the above mentioned algorithms we will try to restore the original image from the degraded image.

\section{b) Basic Parameter}

\section{b1.) MSE (Mean square Error):-}

MSE is defined as the some sort of average or sum (or integral) of square of the error between two image.

Where: (I, J) is the original image

$$
\mathrm{MSE}=\mathrm{I} /(\mathrm{N} * \mathrm{M}) * \mathrm{sum}_{-}\left\{\mathrm{I}, \mathrm{J}\left(\mathrm{X}_{-}\{\mathrm{I}, \mathrm{J}\}-\mathrm{Y}_{-}\{\mathrm{I}, \mathrm{J}\}\right\}\right)^{2}
$$

$\mathrm{Y}(\mathrm{I}, \mathrm{J})$ is the decompressed image

$\mathrm{M}, \mathrm{N}$ is the dimensions of the image

\section{b2.) Peak Signal to Noise Ratio (PSNR):-}

PSNR is defined as the ratio between signal variance and reconstruction error variance:-

$$
\text { PSNR }=20 . \log _{10}\left(\max _{1}\right)-10 . \log _{10}(\mathrm{MSE})
$$

Here: mean squared error (MSE)

$\operatorname{Max}_{1}$ is the maximum possible pixel value of the image 
b3.) RMSE (root mean square error):- RMSE is defined as the square root of mean square error.

$$
\operatorname{RMSE}=\sqrt{ } \mathrm{MSE}
$$

b4.) MAE (maximum absolute error):- MAE is defined as the maximum absolute value, the difference between original image and degraded image

$$
\operatorname{Max}(\operatorname{abs}(\mathrm{I} 1(:)-\mathrm{I} 2(:))
$$

\section{EXPERIMENTS VERIFICATIONS}

All the Implementation work in done in MATLAB 7.5.First of the entire original image is degraded using a degradation function. The degraded image is then deblurred using all of the above deconvolution wiener filter (FFT) techniques, following are tabular results obtained after the comparison:

Table 1. For Different Gaussian Noise Variance apply on PNG Image format

\begin{tabular}{|l|l|l|l|l|l|l|}
\hline GAUSSIAN NOISE & \multicolumn{2}{l|}{ DECONVOLUTION WIENER FILTER USING FFT } & MAE \\
\hline IMAGE & $\begin{array}{l}\text { NOISE } \\
\text { VARIANCE }\end{array}$ & MSE & PSNR & RMSE & 0.6618 \\
\hline PNG & 0.01 & 0.0019 & 75.3329 & 0.0438 & 0.6367 \\
\hline PNG & 0.02 & 0.0020 & 75.2189 & 0.0444 & 0.6326 \\
\hline PNG & 0.03 & 0.0021 & 74.9965 & 0.0455 & 0.6254 \\
\hline PNG & 0.04 & 0.0022 & 74.6678 & 0.0473 & \\
\hline
\end{tabular}

According to Table 1, this work makes a GAUSSIAN NOISE applied on PNG file format using Deconvolution Wiener filter (FFT) Algorithm. A Noise Variance comparison is done on the basis of various performance parameter like PSNR(Peak Signal to Noise Ratio),MSE(Mean Square Error),RMSE(Root Mean Square Error),MAE(Maximum Absolute Error ).

Table 2. For Different Speckle Noise Variance apply on PNG Image format

\begin{tabular}{|l|l|l|l|l|l|}
\hline \multicolumn{3}{|c|}{ SPECKLE NOISE } & \multicolumn{2}{l|}{ DECONVOLUTION WIENER FILTER USING FFT } \\
\hline IMAGE & $\begin{array}{l}\text { NOISE } \\
\text { VARIANCE }\end{array}$ & MSE & PSNR & RMSE & MAE \\
\hline PNG & 0.01 & 0.0017 & 75.8904 & 0.0411 & 0.6030 \\
\hline PNG & 0.02 & 0.0018 & 75.6713 & 0.0421 & 0.6288 \\
\hline PNG & 0.03 & 0.0019 & 75.4905 & 0.0430 & 0.6426 \\
\hline PNG & 0.04 & 0.0019 & 75.3267 & 0.0438 & 0.6732 \\
\hline
\end{tabular}

According to Table 2, this work makes a SPECKLE NOISE applied on PNG file format using Deconvolution Wiener filter (FFT) Algorithm. A Noise Variance comparison is done on the basis of various performance parameter like PSNR(Peak Signal to Noise Ratio),MSE(Mean Square Error),RMSE(Root Mean Square Error),MAE(Maximum Absolute Error ). 
Table 3. For Different Salt \& Pepper Noise Variance apply on PNG Image format

\begin{tabular}{|l|l|l|l|l|l|l|}
\hline \multicolumn{3}{|c|}{ SALT \& PEPPER NOISE } & \multicolumn{3}{l|}{ DECONVOLUTION WIENER FILTER USING FFT } \\
\hline IMAGE & $\begin{array}{l}\text { NOISE } \\
\text { VARIANCE }\end{array}$ & MSE & PSNR & RMSE & MAE \\
\hline PNG & 0.01 & 0.0017 & 75.8477 & 0.0413 & 0.5833 \\
\hline PNG & 0.02 & 0.0018 & 75.6029 & 0.0425 & 0.6064 \\
\hline PNG & 0.03 & 0.0019 & 75.4145 & 0.0434 & 0.6131 \\
\hline PNG & 0.04 & 0.0020 & 75.1916 & 0.0445 & 0.6561 \\
\hline
\end{tabular}

According to Table 3, this work makes a SALT \& PEPPER NOISE applied on PNG file format using Deconvolution Wiener filter (FFT) Algorithm. A Noise Variance comparison is done on the basis of various performance parameter like PSNR(Peak Signal to Noise Ratio),MSE(Mean Square Error),RMSE(Root Mean Square Error),MAE(Maximum Absolute Error ).

Original image

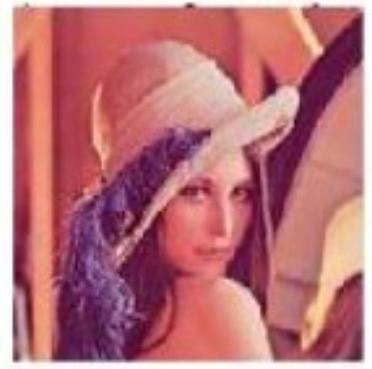

BlurredNoisy

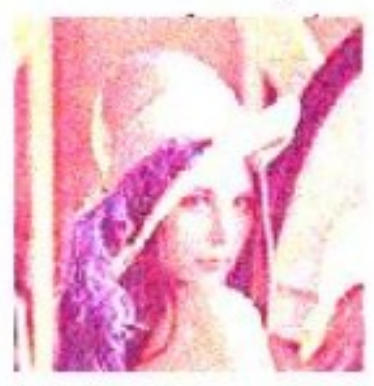

Blur image

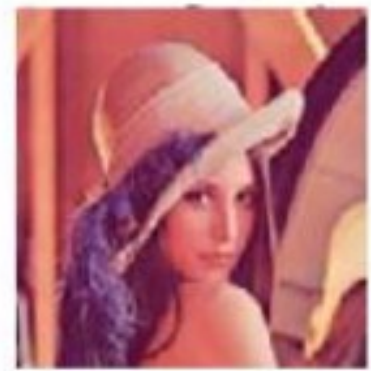

Gaussian noise

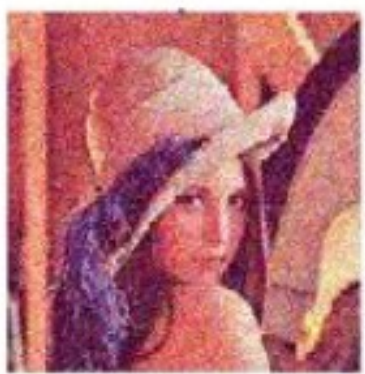

Fig.4. IMAGE IN PNG USED GAUSSIAN NOISE 
Original image

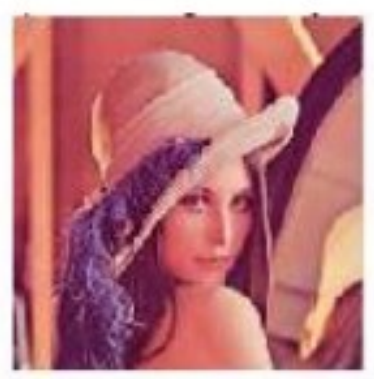

Blur image

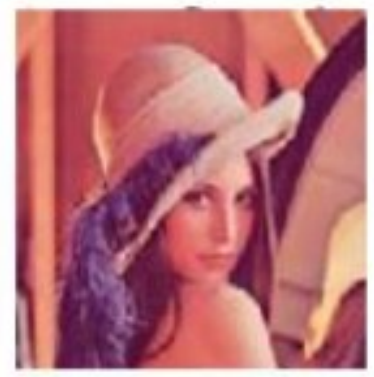

Speckle noise

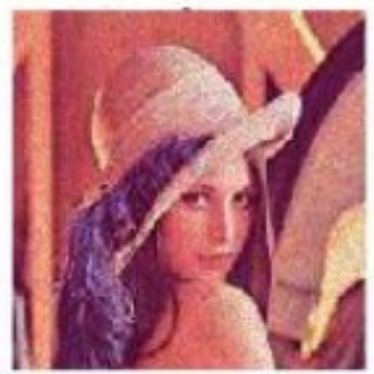

BlurredNoisy

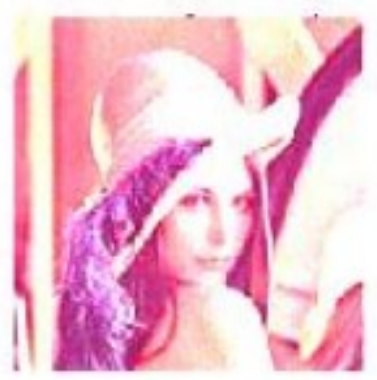

Deblur image using FFT

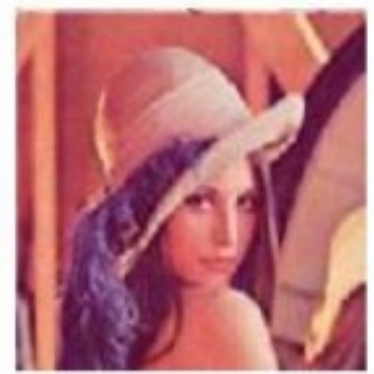

Fig.5. IMAGE IN PNG USED SPECKLE NOISE

Original image

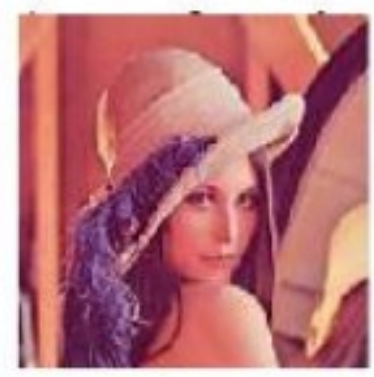

Blur image

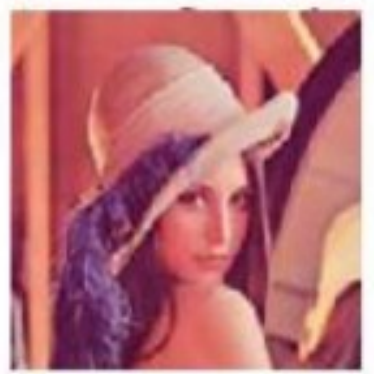

salt \& pepper noise

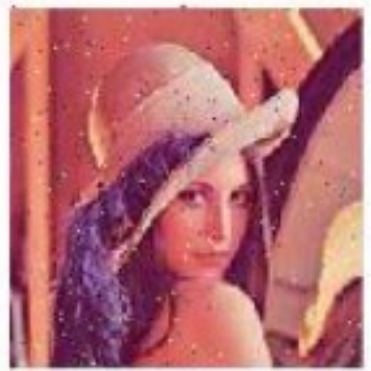

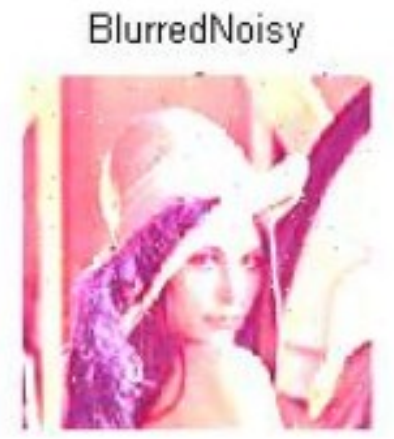

Deblur image using FFT

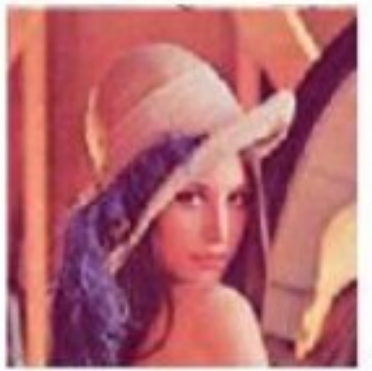

Fig.6. IMAGE IN PNG USED SALT \& PEPPER NOISE 

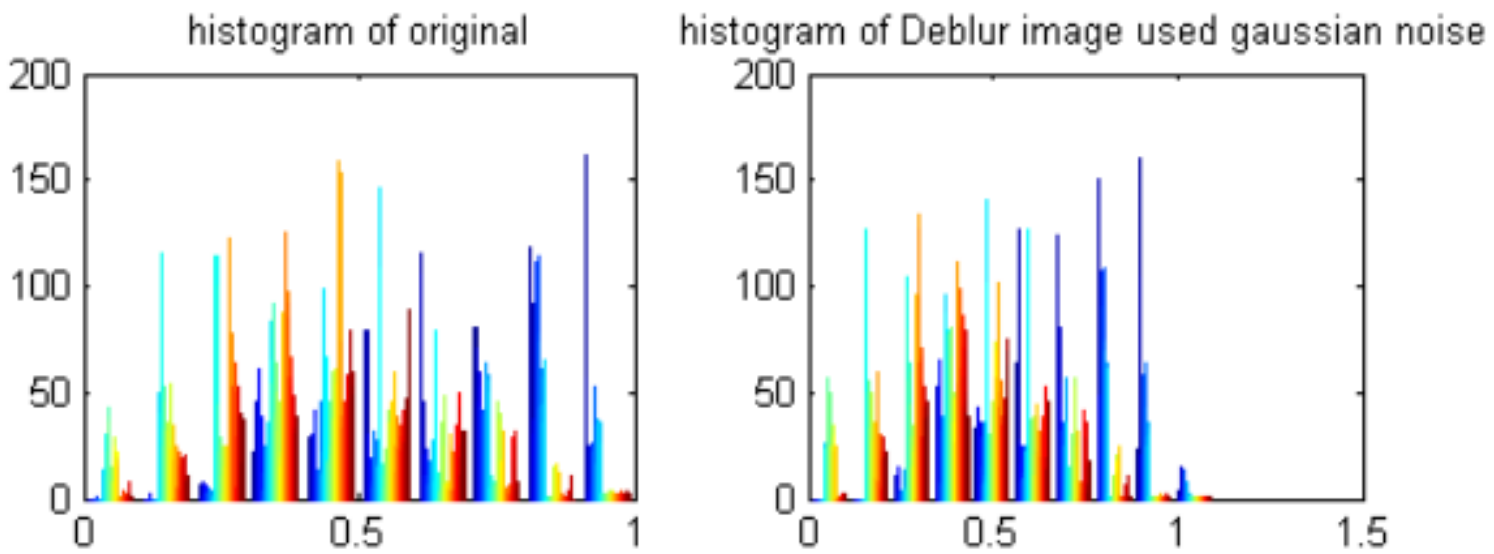

Fig.7. Histogram representation of PNG Image used Gaussian Noise
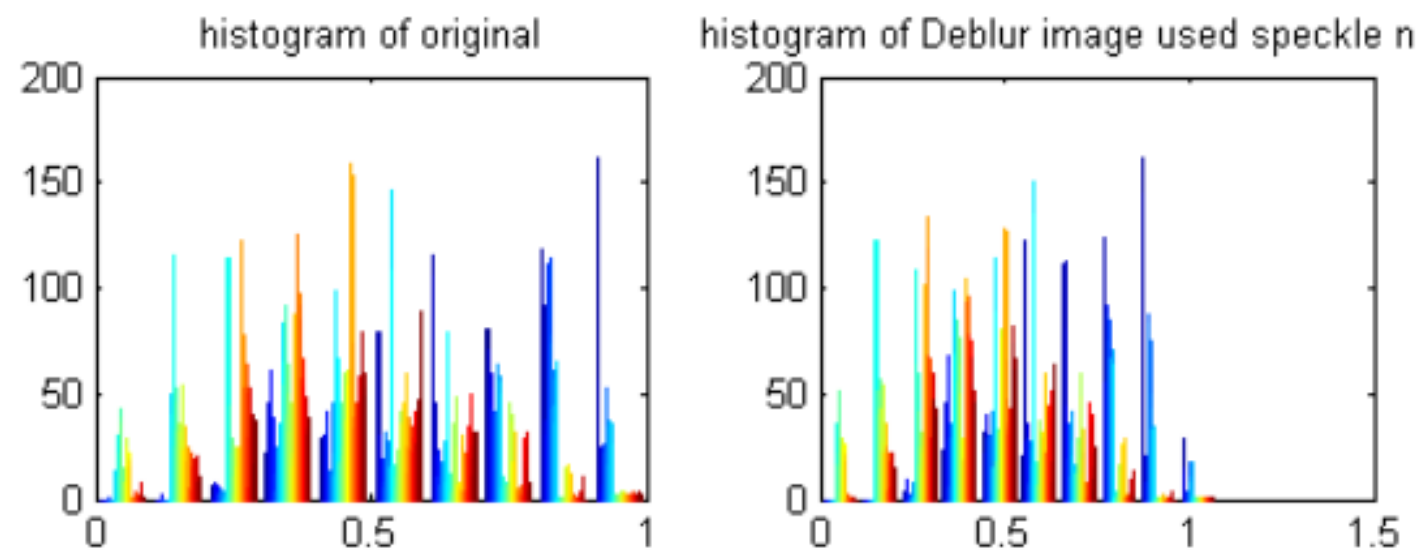

Fig.8. Histogram representation of PNG Image used Speckle Noise

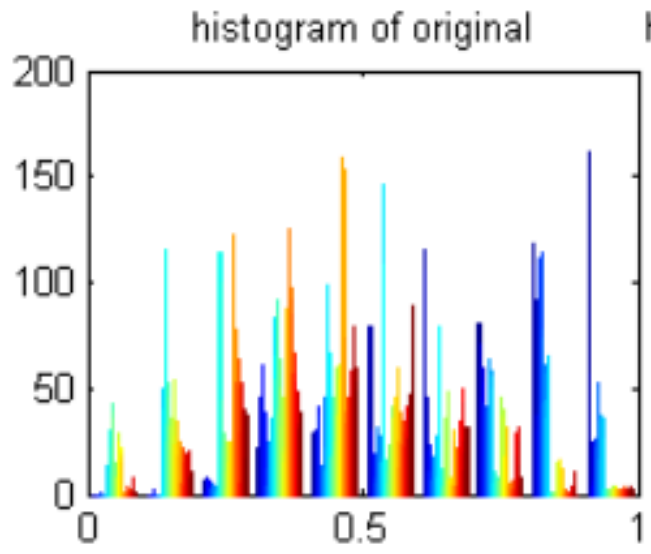

histogram of Deblur image used salt \& pepper noise

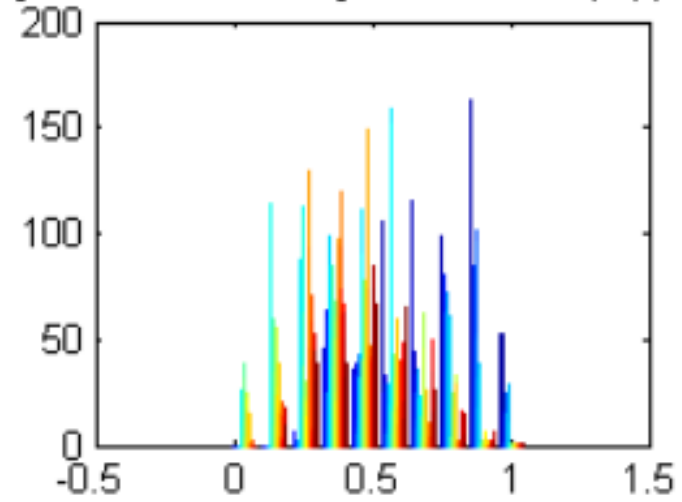

Fig.9. Histogram representation of PNG Image used Salt \& Pepper Noise

The MSE value of GAUSSIAN NOISE is 0.0019,PSNR value of 75.3329 ,RMSE value of 0.0438 ,andMAE value of 0.6618 . The MSE value of SPECKLE NOISE is 0.0017,PSNR value of 75.8904 ,RMSE value of 0.0411 ,andMAE value of 0.6030.The MSE value of SALT \& PEPPER NOISE is 0.0017 ,PSNR value of 75.8477 ,RMSE value of 0.0413 , and MAE value of 0.5833 . Then MAE according to result "SALT \& PEPPER NOISE" is give the better results of png image format.because MAE find the maximum absolute value, the difference between original image and degraded image. Wiener deconvolution is a fast deconvolution algorithm. It is an interesting deconvolution tool that can be very useful to process any kind of image with relatively high signal to noise ratio. Wiener deconvolution is particularly suitable for lunar and planetary imaging. Due to minor optical aberrations or slight tracking errors to quickly find and test PSF. 


\section{REFERENCES}

[1] Shen-Chuan Tai and Shin-Ming yang."'A fast method for image noise estimation using laplacian operator and adaptive edge detection. In Communications”, Control and 1077(1081), 2008.

[2] T. Kobayashi, T.Shimamura, T.Hosoya and Y.Takahashi,"Restoration from Image Degraded by White Noise Based on Iterative Spectral Subtraction Method", IEEE International Symposium on Circuits and Systems, pp.6288-6271, 2005.

[3] Ramys,S.;Mercy Christial,T,'Restoration of Blurred Images using Blind Deconvolution lgorithm,'IEEE,on Emerging Trends in Electrical and Computer Technology(ICETECT),pp.496499,2011.

[4] Dong-Dong Cao, ping Guo, "Blind image restoration based on wavelet analysis,"IEEE, Machine Learning and Cybernetics, pp.-4977, 2005. [5]“International Journal for Science and Emerging Technology with latest Trends"2(1):7-14-(2012).

[6] Dong-Dong Cao, Ping Guo,"Blind Image restoration based on wavelet analysis,'IEEE, Machine Learning and Cybernetics, pp.5977-5982, 2005.

[7] Jiang Ming wang Ge,'Development of blind image deconvolution and its application”, Journal of X-Ray Science and Technology, IOS press, 11(2003), pp.13-19.

[8] Kundur Deepa, Hatzinakos,'Blind Image Deconvolution", IEEE Signal processing Magazine, 13(6) May (1996), pp.43-64. 